\title{
Matrix Metalloproteinase-9 and Augmentation Index are Reduced with an 8-Week Green-Exercise Walking Programme
}

\author{
Jane ES Thompson ${ }^{1 *}$, Richard Webb ${ }^{1}$, Paul Hewlett ${ }^{1}$, David Llewellyn ${ }^{2}$, Barry J McDonnell ${ }^{1}$ \\ ${ }^{1}$ Cardiff School of Health Sciences, Cardiff Metropolitan University, Wales, UK \\ ${ }^{2}$ Groundwork Wales Ltd, Treforest, Wales, UK
}

\begin{abstract}
Objectives: MMP-9 is involved in degrading the Extracellular Matrix (ECM); specifically elastin, which provides elasticity to the arterial wall. Elastin degradation and restructuring of the ECM results in increased vascular remodelling and arterial stiffness. Conversely, exercise improves age-related vascular stiffening. Therefore, this study aimed to investigate whether participation in a green-exercise programme affects vascular haemodynamics and mRNA expression of MMP-9.
\end{abstract}

Methods: Thirty-six healthy, sedentary individuals ( $44 \pm 2 y r s ;$ not taking any cardiovascular-acting medication) joining a moderate-intensity, aerobic green-exercise programme, were recruited. At baseline and 8-weeks into the programme, physical activity (measured in weekly MET-minutes [IPAQ]), supine Mean Arterial Blood Pressure (MAP), Augmentation Index (Alx) and aortic Pulse Wave Velocity (aPWV) data were collected and blood samples were obtained. Leukocytic MMP-9 mRNA expression (RT-PCR) and plasma protein levels (ELISA) were analysed; Alx and aPWV were measured via applanation tonometry (SphygmoCor, Atcor Medical, Australia).

Results: The cohort was split into those who adhered $(n=17)$ and did not adhere $(n=19)$ to the programme. MMP-9 expression, MAP and Alx all decreased significantly in the exercise-adherent group (cf. the non-adherent group), while significant correlations were seen between: (i) $\Delta \mathrm{MMP}-9$ expression and $\triangle \mathrm{MET}$-minutes/wk; (ii) $\triangle \mathrm{MMP}-9$ expression and $\triangle \mathrm{Alx}$; (iii) $\triangle \mathrm{Alx}$ and $\triangle \mathrm{MET}$-minutes/wk $(\mathrm{P}<0.05$ in all cases). aPWV did not change significantly between the groups.

Conclusions: These findings suggest that exercise-induced down-regulation of MMP-9 may contribute to reduced ECM degradation and therefore ameliorate vascular remodelling. Additional studies are needed to explore these findings further; however, these data may provide a biomolecular mechanism for aerobic exercise's ability to delay age-related increases in arterial stiffening.

Keywords: Matrix metalloproteinase-9; Vascular remodelling; Arterial stiffness; Wave reflection; Aerobic exercise; Green-Exercise

Abbreviations : Ad: Adherent; AIx: Augmentation Index; AP-1: Activator Protein-1; aPWV: Aortic Pulse Wave Velocity; BP: Blood Pressure; DBP: Diastolic Blood Pressure; cDNA: complementary DNA; $\mathrm{C}^{\mathrm{T}}$ : Threshold Cycle; ECM: Extracellular Matrix; ELISA: EnzymeLinked Immunosorbent Assay; GAPDH: Glyceraldehyde 3-Phosphate Dehydrogenase; HDL: High Density Lipoprotein; IPAQ: International Physical Activity Questionnaire; LDL: Low Density Lipoprotein; MAP: Mean Arterial Pressure; MET: Metabolic Equivalent of Task; MMP-9: Matrix Metalloproteinase-9; mRNA: messenger RNA; Nfkb: Nuclear Factor Kappa Beta; Nfkb-RE: Nuclear Factor Kappa Beta Response Element; Non-Ad: Non-Adherent; PP: Pulse Pressure; SBP: Systolic Blood Pressure; SES: Socio-Economic Status; PPAR $\gamma$ : Peroxisome Proliferator-Activated Receptor Gamma; PPRE: Peroxisome Proliferator Response Element; q-PCR: Quantitative Real Time PCR; RCF: Relative Centrifugal Force; RSG: Rosiglitazone; RT-PCR: Reverse Transcription Polymerase Chain Reaction; WC: Waist Circumference

\section{Introduction}

One of the main risk factors associated with increased Cardiovascular (CV) risk is increased arterial stiffness which occurs naturally with aging but prematurely in $\mathrm{CV}$ disease states such as diabetes [1] and as a consequence of leading physically inactive lifestyles [2-4]. Arterial stiffness is partly determined by the elastic properties of the arterial wall, with increased vascular remodelling being associated with increased stiffness and CV risk [5-8]. Vascular remodelling occurs due to a breakdown of the local Extracellular Matrix (ECM), reorganisation of the ECM and smooth muscle cell migration to the intima [9-11]. Specifically the breakdown and reorganisation of the ECM disrupts the ratio of two of its proteinaceous components: collagen (which maintains the structure of the arterial wall by providing tensile strength) and elastin (which aids in maintaining the elasticity of the arterial wall). As a result of increased elastin degradation and the associated increased vascular remodelling, the compliant nature of the vessel is diminished, meaning that the arterial wall is less able to expand and recoil in response to changing blood pressures, and hence loses its capacity to buffer flow and pressure [12]. Consequently, arterial blood pressure, cardiac afterload and therefore cardiac work are all increased [13]

Enzymes of the Matrix Metalloproteinase (MMP) family have been identified as playing a major role in the degradation and reorganisation of the ECM. MMPs are zinc dependent endopeptidases that, once secreted into the ECM, are capable of cleaving components of the

*Corresponding author: Jane ES Thompson, Centre for Biomedical Sciences, Cardiff School of Health Sciences, Cardiff Metropolitan University, Western Avenue, Cardiff, CF5 2YB, United Kingdom, Tel: +44 (2920) 416457; Fax: +44 (2920) 416982; E-mail: jthompson@cardiffmet.ac.uk

Received August 29, 2013; Accepted September 30, 2013; Published Octobe 03, 2013

Citation: Thompson JES, Webb R, Hewlett P, Llewellyn D, McDonnell BJ (2013) Matrix Metalloproteinase-9 and Augmentation Index are Reduced with an 8-Week Green-Exercise Walking Programme. J Hypertens 2: 127. doi:10.4172/21671095.1000127

Copyright: (C) 2013 Thompson JES, et al. This is an open-access article distributed under the terms of the Creative Commons Attribution License, which permits unrestricted use, distribution, and reproduction in any medium, provided the original author and source are credited. 
Citation: Thompson JES, Webb R, Hewlett P, Llewellyn D, McDonnell BJ (2013) Matrix Metalloproteinase-9 and Augmentation Index are Reduced with an 8-Week Green-Exercise Walking Programme. J Hypertens 2: 127. doi:10.4172/2167-1095.1000127

ECM [14]. They are regulated at the level of gene transcription, posttranslational activation of zymogens and interaction of secreted MMPs with inhibitors such as tissue inhibitor of metalloproteinases [15]. As an elastin-degrading enzyme, MMP-9 has received particular attention in the context of vascular structure and functioning, with elevated MMP9 levels being associated with increased aortic pulse wave velocity (aPWV) [16] the current gold-standard method of measuring arterial stiffness [17].

It has been demonstrated many times that exercise programmes and physically active lifestyles are associated with improvements in CV risk via reductions in risk factors such as arterial stiffness $[2,18,19]$. Despite these benefits, $80 \%$ of the population do not undertake sufficient exercise to gain the associated effects [20]. A useful tool for engaging a greater proportion of the population in exercise may be green-exercise; that is, any exercise that takes place in a relatively natural setting [21]. Previously, acute bouts of exercise whilst viewing a natural scene resulted in greater reductions in mean arterial pressure and self-esteem than exercising without a visual stimulus [21]. The symbiotic effect of exercise and the natural environment is thought to amplify the beneficial effects of exercise due to the natural environment not requiring direct and effortful attention and therefore providing an opportunity to recover from mental stress that is induced in urban and built-up environments [22]. Green-exercise is free and more easily accessible than traditional gym-based exercise and hence overcomes many reported barriers to exercise [23]. However, the impact of a green-exercise programme on markers of health including CV risk has not previously been assessed.

The associated molecular mechanisms driving these exerciseassociated improvements are not fully understood. Previous reports have linked exercise to reductions in MMP-9 levels, but have been limited to resistance training programmes in men only or exerciseplus-diet interventions in men with metabolic syndrome and children with atherosclerotic risk factors [24-26]. Similarly, Kadaglou et al. demonstrated that in a diabetic population, a 16-week exercise programme resulted in decreased plasma levels of MMP-9 [27].

However, these previous studies did not assess vascular haemodynamics, and therefore could not determine whether the reductions in MMP-9 were associated with changes in wave reflection or aPWV. Also, to our knowledge, the effects of aerobic green-exercise programmes on MMP-9 mRNA expression and markers of CV risk in healthy individuals have not previously been assessed.

Therefore, the aims of this study were to test the hypotheses that;

1. Moderate-intensity, aerobic green-exercise is associated with reductions in Augmentation Index (AIx) and the expression (at both the mRNA and protein levels) of the gene encoding the elastin-degrading enzyme, namely MMP-9.

2. Moderate-intensity, aerobic green-exercise elicits beneficial effects on markers of arterial function and $\mathrm{CV}$ risk via its impact on vascular haemodynamics (and possibly its effects on MMP-9).

\section{Methods}

\section{Study population}

36 participants aged 44 ( \pm 2SE) years old, who signed up to begin regular participation in a green-exercise walking programme, were recruited. To meet the inclusion criteria, participants were required to be sedentary (defined as not taking part in any regular or structured exercise in the previous six months [28]), healthy (defined as having no chronic illnesses or taking cardiovascular-acting medication), not pregnant (or likely to become pregnant), over the age of 18 years, and able to provide written consent.

Participants signed up to their chosen green-exercise walking programme independently of the research project. Before commencing their programme, they were assessed in terms of suitability for the project, and were then invited to participate in the project. The walking groups from which participants were recruited were based in rural/countryside areas, were sited in areas of low Socio-Economic Status (SES), and were set up as affiliates of Groundwork Wales Ltd; an environmental regeneration body aiming to build sustainable communities across Wales and the UK.

\section{Ethics}

All research was conducted in accordance with the Declaration of Helsinki. Ethical approval was obtained from the Cardiff School of Health Sciences Research Ethics Committee of Cardiff Metropolitan University. Written informed consent was obtained from each participant prior to the collection of data.

\section{Green-exercise}

The study was carried out in the south Wales valleys, an area of high social and economic deprivation and low SES, low physical activity levels and some of the worst health statistics in the UK and Europe [29]. Community-based green-exercise walking group initiatives (as facilitated by Groundwork Wales Ltd in the present case) have been employed as a method of combating the SES-associated low physical activity and poor health in these areas, by engaging people with exercising in their local area/environment. On average, participants on the green-exercise programmes took part in two walks per week, for 45 minutes each. Intensity of the walks was approximately $4.3 \pm 0.3 \mathrm{SE}$ METs, as determined via accelerometers (BodyMedia SenseWear, PA, USA), and the intensity was therefore considered moderate [30]. Over the 8-week period, registers of attendance were taken for each walk in order to measure the compliance of each participant to the exercise programme.

\section{Experimental sampling procedures}

Participants underwent two consultations; one at baseline, when participants were still sedentary; the second took place eight weeks into the green-exercise programme. During each consultation, participants completed the International Physical Activity Questionnaire (IPAQ) in order to assess their weekly physical activity levels. Consultations took place in the morning and under fasting conditions (no consumption of food on the day of testing, no consumption of tobacco products, alcohol or caffeine for 12 hours prior to testing); also, participants had not carried out any strenuous exercise in the previous 24 hours. Participants completed a dietary questionnaire during each consultation in order to determine whether any changes in diet occurred over the period of the intervention.

Anthropometric measurements: During each consultation, participants had height, weight, waist circumference and Body Mass Index (BMI) measured. Following a seated rest period of 10 minutes, brachial Blood Pressure (BP) was measured (Omron Corporation, Ill, USA) on the non-dominant arm (according to BHS guidelines) [31]. Blood pressure readings were used to calculate mean arterial pressure (MAP) using the formula: MAP $=$ diastolic $\mathrm{BP}+0.333$ (systolic BPdiastolic BP) [32]. 
Citation: Thompson JES, Webb R, Hewlett P, Llewellyn D, McDonnell BJ (2013) Matrix Metalloproteinase-9 and Augmentation Index are Reduced with an 8-Week Green-Exercise Walking Programme. J Hypertens 2: 127. doi:10.4172/2167-1095.1000127

Vascular haemodynamic measurements: Non-invasive measures of aPWV (a measure of large artery stiffness) and AIx (a measure of wave reflection) were recorded using applanation tonometry (Atcor Medical, Sydney, Australia), according to recommended guidelines [17]. AIx was recorded at the right radial pulse and pulse wave analysis of the central aortic pulse waveform was carried out using SphygmoCor software (Atcor Medical).

Following a supine rest period of 5 minutes, BP was measured before measuring aPWV, which measures the velocity of the pulse pressure wave as it travels along the aorta during each cardiac cycle. Carotid and femoral pulses were palpated, and the distance of each from the suprasternal notch was measured in order to determine the pulse wave path length. Sequential ECG-gated pressure waveforms of the right carotid and femoral arteries were obtained in order to assess aPWV. The R-wave of the ECG was used as a reference frame for each cardiac cycle and the SphygmoCor device automatically calculated pulse wave transit time as the time delay between the carotid and femoral 'foot' waveforms. aPWV was then calculated as the 'distance: transit time' ratio, expressed in metres per second $(\mathrm{m} / \mathrm{s})$. aPWV data were normalised to the mean arterial pressure of the cohorts at baseline and at 8-weeks.

AIx measures the augmentation of aortic pressure during late systole (P2) compared to early systole (P1), with the difference (augmentation pressure) being expressed as a percentage of aortic pulse pressure. Thus, AIx, a measure of wave reflection, is considered a surrogate and indirect measure of arterial stiffness in which, as vascular remodelling increases and sites of impedance mis-match become closer to the heart, the forward travelling wave that is released following left ventricular ejection is reflected back to the heart earlier [17]. This causes the wave to arrive at the proximal aorta during late systole rather than early diastole, thus augmenting late systolic pressure (P2).

Molecular biology laboratory methods: $15 \mathrm{mls}$ of fasting, venous blood from the antecubital vein was collected. Blood was collected into SST, lithium-heparin and fluoride-oxalate blood collection tubes (Vacuette, Greiner bio-one, Gloucestershire, UK). Tubes were gently inverted eight times immediately following blood collection. The SST and fluoride-oxalate blood tubes were spun (10 minutes, $400 \mathrm{RCF}$, $4^{\circ} \mathrm{C}$ ) in order to obtain serum and plasma samples, respectively, before storing aliquots at $-80^{\circ} \mathrm{C}$ for use in subsequent analyses (see Biochemical analysis section). The blood from the lithium-heparin tube was gently added (1:1 v: v) onto Histopaque-1077 (Sigma Aldrich, Poole, UK) before centrifugation ( 30 minutes, $400 \mathrm{RCF}, 4^{\circ} \mathrm{C}$ ). Following this spin, plasma was aspirated from the top layer of the resulting mixture and stored at $-80^{\circ} \mathrm{C}$ for use in subsequent ELISA experiments (see ELISA section), while the 'buffy coat' interface (containing a mixed leukocyte sample) was gently aspirated into a fresh tube, mixed with $10 \mathrm{ml}$ of phosphate buffered saline, spun and washed twice $(2 \times 10$ minutes, 250 $\mathrm{RCF}, 4^{\circ} \mathrm{C}$ ). The supernatant was removed, and the pellet re-suspended in $1 \mathrm{ml}$ of Trizol Tri-reagent (Invitrogen, Paisley, UK) for storage at $-80^{\circ} \mathrm{C}$ for use in subsequent RT-PCR analyses (see RT-PCR section).

Biochemical analysis: Total-cholesterol, high density lipoprotein
(HDL) and triglycerides were measured in the serum samples and glucose was measured in the plasma samples, using an ILab 300 Plus analyser (Instrumentation Laboratory, Warrington, UK). Low density lipoprotein (LDL) was calculated from the total-cholesterol and HDL using the Friedwald equation [33].

ELISA: Plasma MMP-9 levels were determined as per the manufacturer's guidelines using Quantikine Human MMP-9 ELISA experiments (R\&D Systems, Abingdon, UK); this ELISA is designed to measure MMP-9's active forms (Package Insert accompanying ELISA kit from R\&D Systems) [34].

RT-PCR: RNA was extracted from the mixed leukocyte samples via the phenol/chloroform extraction method, and converted to cDNA (High Capacity cDNA Conversion Kit; Invitrogen, Paisley, UK), as per the manufacturer's guidelines. Only samples with yields above $15 \mathrm{ng} / \mu \mathrm{l}$ RNA, with a 260/280 ratio of 1.8 , and 260/280 ratio of between 2.0 and 2.2 (Nanodrop ND-1000 Spectrophotometer; Thermo Scientific, DE, USA) were used in subsequent analyses.

Using the derived cDNA samples, q-PCR experiments were run using an Applied Biosystems 7500 real-time PCR system (Applied Biosystems, Warrington, UK) in order to measure the expression of the gene of interest; MMP-9. GAPDH was used as the endogenous reference gene. Primer oligonucleotides (Sigma Aldrich, Poole, UK) were designed using OligoPerfect Designer (Invitrogen, Paisley, UK), as shown in Table 1. $\mathrm{C}^{\mathrm{T}}$ values were determined fluorimetrically via the use of SYBR Green PCR Master Mix (Applied Biosystems, Warrington, $\mathrm{UK})$.

The relative quantification of the target genes in the mixed leukocytes was calculated via the $2^{-\Delta \Delta \mathrm{CT}}$ formula, in which $\Delta$ represents the difference between $\mathrm{C}^{\mathrm{T}}$ values for baseline and 8-week samples and $\Delta \mathrm{C}^{\mathrm{T}}$ represents the difference between $\mathrm{C}^{\mathrm{T}}$ values for the gene of interest and GAPDH in each case.

\section{Bioinformatics analysis}

Sequence analyses and alignments were performed using DNASTAR software (Lasergene, version 7; DNASTAR Inc., WI, USA). All sequences were obtained from National Center for Biotechnology Information databases (http://www.ncbi.nlm.nih.gov/).

\section{Statistical methods}

All data are presented as mean $\pm \mathrm{SE}$, unless otherwise stated. Our analytical approach was based on independent sample $t$ tests comparing the delta change from baseline to 8-weeks, between the two groups. Correlation analyses were also performed using Pearson's method. Statistical significance was set a priori to $\mathrm{P}<0.05$; other results were reported as 'NS'. All statistical analyses were performed using SPSS for Windows, version 20 (SPSS Inc., Ill, USA). Analysis of the ELISA data was carried out using Graph Pad Prism 5 software (GraphPad Software Inc., CA, USA).

\section{Results}

Walk registers and IPAQ MET-minutes revealed that 19 of the 36

\begin{tabular}{|c|c|c|c|}
\hline Gene (accession no.) & Forward primer (5'-3') & Reverse primer (5'-3') \\
\hline MMP-9 (NM_004994.2) & TTGACAGCGACAAGAAGTGG & CCCTCAGTGAAGCGGTACAT & Amplicon (bp) \\
\hline GAPDH (NM_002046.3) & CATTGACCTCAACTACATG & TCTCCATGGTGGTGAAGAC & 148 \\
\hline
\end{tabular}

Table 1: Primer sequences used to analyse genes of interest using RT-PCR. 
Citation: Thompson JES, Webb R, Hewlett P, Llewellyn D, McDonnell BJ (2013) Matrix Metalloproteinase-9 and Augmentation Index are Reduced with an 8-Week Green-Exercise Walking Programme. J Hypertens 2: 127. doi:10.4172/2167-1095.1000127

Page 4 of 8

\begin{tabular}{|c|c|c|c|}
\hline Baseline characteristics & Adherent group & Non-adherent group & $P$ value \\
\hline$N$ & 17 & 19 & NS \\
\hline Female (n) & 17 & 17 & NS \\
\hline Age (years) & $42 \pm 4$ & $45 \pm 3$ & NS \\
\hline WC (cm) & $84 \pm 2$ & $85 \pm 3$ & NS \\
\hline Weight (kg) & $73 \pm 2$ & $70 \pm 3$ & NS \\
\hline BMI $\left(\mathrm{kg} / \mathrm{m}^{2}\right)$ & $26.8 \pm 0.8$ & $26.4 \pm 1.1$ & NS \\
\hline IPAQ (MET-Minutes/wk) & $218 \pm 50$ & $356 \pm 67$ & NS \\
\hline SBP $(m m H g)$ & $122 \pm 3$ & $115 \pm 2$ & NS \\
\hline DBP $(\mathrm{mmHg})$ & $74 \pm 3$ & $74 \pm 2$ & NS \\
\hline PP (mmHg) & $48 \pm 4$ & $41 \pm 2$ & NS \\
\hline MAP $(\mathrm{mmHg})$ & $90 \pm 2$ & $88 \pm 2$ & NS \\
\hline Alx at HR $75(\%)$ & $16.6 \pm 3.4$ & $19.8 \pm 2.8$ & NS \\
\hline $\operatorname{aPWV}(\mathrm{m} / \mathrm{s}) \#$ & $6.7 \pm 0.2$ & $6.9 \pm 0.2$ & NS \\
\hline T-Cholesterol (mmol/L) & $4.9 \pm 0.4$ & $4.9 \pm 0.3$ & NS \\
\hline LDL (mmol/L) & $3.1 \pm 0.4$ & $3.0 \pm 0.2$ & NS \\
\hline HDL (mmol/L) & $1.9 \pm 0.2$ & $2.2 \pm 0.2$ & NS \\
\hline Triglycerides (mmol/L) & $0.9 \pm 0.1$ & $1.0 \pm 0.1$ & NS \\
\hline Glucose (mmol/L) & $5.1 \pm 0.2$ & $4.6 \pm 0.1$ & 0.01 * \\
\hline
\end{tabular}

Data are means \pm SE,\# data adjusted for mean arterial pressure.

${ }^{*} P \leq 0.05$ between adherent group and non-adherent group.

WC: Waist Circumference; BMI: Body Mass Index; IPAQ: International Physical Activity Questionnaire; MET: Metabolic Equivalent; wk: Week; SBP: Systolic Blood Pressure; DBP: Diastolic Blood Pressure; PP: Pulse Pressure; MAP: Mean Arterial Pressure; Aix: Augmentation Index; aPWV: aortic Pulse Wave Velocity; LDL: Low Density Lipoprotein; HDL: High Density Lipoprotein; NS: Not Significant.

Table 2: Baseline characteristics of the two study groups.

\begin{tabular}{|c|c|c|c|}
\hline $\begin{array}{c}\Delta \text { Change baseline-8 } \\
\text { weeks }\end{array}$ & Adherent group & Non-adherent group & $P$ value \\
\hline WC (cm) & $-1.2 \pm 0.4$ & $-0.2 \pm 0.7$ & NS \\
\hline Weight (kg) & $+0.01 \pm 0.3$ & $+0.4 \pm 0.3$ & NS \\
\hline BMI (kg/m $)$ & $-0.01 \pm 0.1$ & $+0.1 \pm 0.1$ & NS \\
\hline IPAQ (MET-Minutes/wk) & $+712 \pm 137$ & $-101 \pm 67$ & $<0.001^{* *}$ \\
\hline SBP (mmHg) & $-7 \pm 3$ & $-0 \pm 2$ & NS \\
\hline DBP (mmHg) & $-0 \pm 2$ & $+2 \pm 1$ & NS \\
\hline PP (mmHg) & $-6 \pm 3$ & $-2 \pm 1$ & NS \\
\hline MAP (mmHg) & $-3 \pm 2$ & $+1 \pm 1$ & 0.04 * \\
\hline Alx at HR 75 (\%) & $-4.1 \pm 1.8$ & $+1.9 \pm 2.2$ & 0.04 * \\
\hline aPWV(m/s) \# & $+0.3 \pm 0.2$ & $+0.2 \pm 0.2$ & NS \\
\hline T-Cholesterol (mmol/L) & $-0.2 \pm 0.1$ & $-0.1 \pm 0.1$ & NS \\
\hline LDL (mmol/L) & $-0.1 \pm 0.1$ & $0.0 \pm 0.1$ & NS \\
\hline HDL (mmol/L) & $-0.1 \pm 0.1$ & $0.0 \pm 0.1$ & NS \\
\hline Triglycerides (mmol/L) & $-0.1 \pm 0.2$ & $-0.2 \pm 0.1$ & NS \\
\hline Glucose (mmol/L) & $-0.1 \pm 0.2$ & $+0.4 \pm 0.2$ & NS \\
\hline
\end{tabular}

Data are means \pm SE, \# data adjusted for mean arterial pressure.

${ }^{*} P \leq 0.05$ between adherent group and non-adherent group, ${ }^{* *} P \leq 0.001$ between adherent group and non-adherent group.

WC: Waist Circumference; BMI: Body Mass Index; IPAQ: International Physical Activity Questionnaire; MET: Metabolic Equivalent; wk: Week; SBP: Systolic Blood Pressure; DBP: Diastolic Blood Pressure; PP: Pulse Pressure; MAP: Mean Arterial Pressure; Aix: Augmentation Index; aPWV: aortic Pulse Wave Velocity; LDL: Low Density Lipoprotein; HDL: High Density Lipoprotein; NS: Not Significant.

Table 3: Delta changes from baseline to 8-weeks, for the two study groups, and

the statistical significance of the difference in delta change in each case.

participants had not increased weekly physical activity from baseline, whereas the remaining 17 participants had $(\mathrm{P}<0.001)$. The cohort was therefore split into an exercise adherent group (Ad: $n=17)$, and an exercise non-adherent group (Non-Ad: $n=19$ ).

At baseline, independent sample $t$ tests demonstrated that physical activity levels (as assessed by IPAQ questionnaire), anthropometric,

BP, AIx, aPWV and biochemical data for the two groups were similar, with the only significant difference between them being fasting glucose levels (Table 2).

As expected, the exercise-adherent group had a significantly greater increase in weekly IPAQ MET-minutes over the course of the intervention than the non-adherent group (Ad: $712 \pm 137 \mathrm{v}$. NonAd: $-101 \pm 67$ MET-minutes, $\mathrm{P}<0.001)$. However there were no significant differences between the two groups with regard to changes in weight, waist circumference or BMI from baseline to 8-weeks (Table 3). Therefore, it appears that all observed exercise-associated changes occurred independently of anthropometric measures. Over the period of the intervention, the diet of the participants remained stable and underwent no significant changes (data not shown).

As shown in Figure 1, the adherent group demonstrated a significant decrease in MAP from baseline to 8-weeks, cf. the non-adherent group (Ad: $-3 \pm 2 \mathrm{mmHg}$. Non-Ad: $+1 \pm 1 \mathrm{mmHg}, \mathrm{P}=0.04$ ). Adherence to the exercise programme was also associated with a significant decrease in AIx, compared to the non-adherent group (Ad: $-4.1 \pm 1.8 \%$ v. Non-

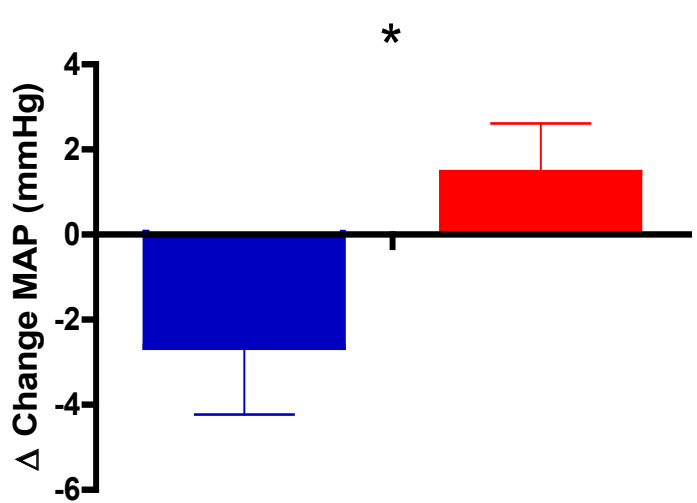

Figure 1: The change in mean arterial pressure between the exercise adherent group and the exercise non-adherent group, from baseline to 8-weeks of a green exercise programme. Mean arterial pressure was calculated following measurement of blood pressure at the brachial artery, following a supine rest period of 5 minutes $\left({ }^{*} P<0.05\right.$; Blue bar; Adherent-group [n=17]; Red bar; Nonadherent group $[\mathrm{n}=19])$. Error bars represent standard error.

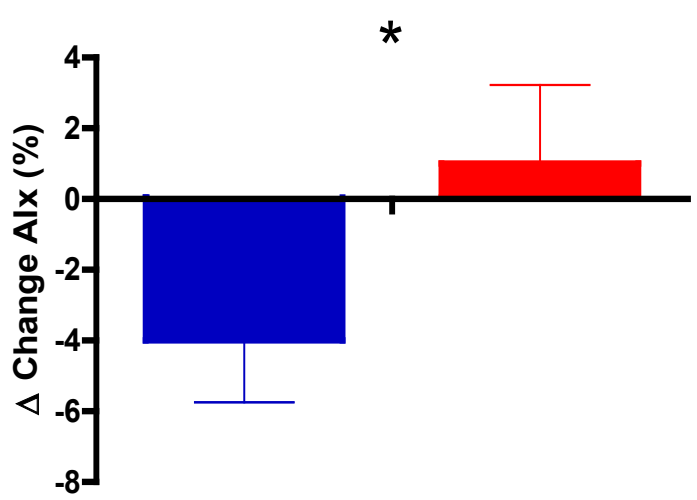

Figure 2: The change in augmentation index between the exercise adherent group and the exercise non-adherent group, from baseline to 8-weeks of a green exercise programme. Augmentation index was measured at the right radial pulse and normalised to a heart rate of $75 \mathrm{bpm}$; pulse wave analysis of the central aortic pulse waveform was carried out using SphygmoCor software (Atcor Medical). ( ${ }^{*} \mathrm{P}<0.05$; Blue bar; Adherent-group [ $\left.\mathrm{n}=17\right]$; Red bar; Nonadherent group [ $\mathrm{n}=19]$ ). Error bars represent standard error. 
Citation: Thompson JES, Webb R, Hewlett P, Llewellyn D, McDonnell BJ (2013) Matrix Metalloproteinase-9 and Augmentation Index are Reduced with an 8-Week Green-Exercise Walking Programme. J Hypertens 2: 127. doi:10.4172/2167-1095.1000127

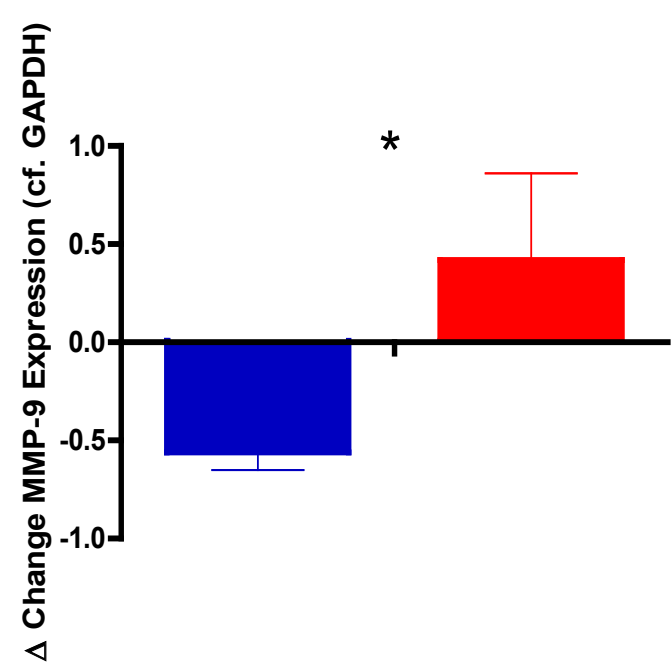

Figure 3: The change in matrix metalloproteinase-9 expression between the exercise adherent group and the exercise non-adherent group, from baseline to 8-weeks of a green exercise programme. MMP-9 mRNA expression was determined using RT-PCR, and is expressed relative to that of GAPDH as an endogenous reference gene $\left({ }^{*} P<0.05\right.$; Blue bar; Adherent-group [n=17]; Red bar; Non-adherent group [ $n=19])$. Error bars represent standard error.

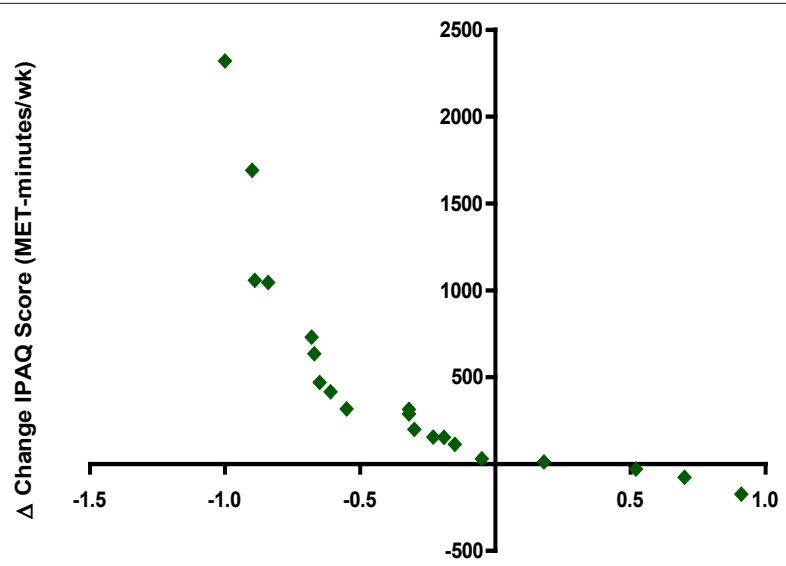

$\Delta$ Change MMP-9 Expression (cf. GAPDH)

Figure 4: The relationship between the changes in IPAQ MET-minutes/wk and matrix metalloproteinase-9 expression recorded between baseline and 8-weeks of a green exercise programme. A statistically significant association between measured changes in MET-minutes (as determined using IPAQ questionnaire responses) and in MMP-9 mRNA expression (as determined using RT-PCR) was identified ( $r=-0.61, P=0.004)$ using Pearson's correlation method.

Ad: $+1.9 \pm 2.2 \%, P=0.04$ ) (Figure 2). Furthermore, these data also illustrated that there was no significant change in aPWV observed in the exercise adherent group compared to the non-adherent group, following the 8-week intervention.

Importantly, adherence to the exercise programme was associated with a significant decrease in the expression of the MMP-9 gene (as assessed by RT-PCR); this was a significantly different response from that seen in the non-adherent group (Ad: $-0.56 \pm 0.09$ v. Non-Ad: + $0.42 \pm 0.44, \mathrm{P}=0.03$ ) (Figure 3 ). Given the extracellular site of action MMP-9 [35], ELISA measurements determining levels of secreted MMP-9 within the plasma were also carried out; no significant difference between the baseline-to-8-week changes for the adherent group and the non-adherent group were observed, although there appeared to be a greater reduction in MMP-9 levels in the adherent group (Ad: -28.4 \pm $21.1 \mathrm{ng} / \mathrm{mL}$ v. Non-Ad: $-20.5 \pm 6.0 \mathrm{ng} / \mathrm{mL}, \mathrm{P}=\mathrm{NS}$ ) and when change in plasma MMP-9 levels was correlated against change in MMP-9 mRNA expression, a significant and positive relationship between the two was observed $(r=0.64, P=0.002$; See Figure, Supplemental Digital Content 1 , which demonstrates the significant and positive relationship between the change in plasma MMP-9 levels and the change in MMP-9 mRNA expression) [35].

Correlation analyses were performed in an attempt to investigate the relationships between the above effects. When change in MMP-9 expression was correlated against change in weekly MET-minutes, a significant inverse relationship between the two parameters was observed $(\mathrm{r}=-0.61, \mathrm{P}=0.004)$ (Figure 4$)$. The change in the expression of MMP-9 mRNA was correlated against the change in AIx, and a significant positive relationship between the two was observed $(r=0.87$, $\mathrm{P}<0.001$ ) (Figure 5). Furthermore, a significant inverse relationship was observed between AIx and weekly MET-minutes $(\mathrm{r}=-0.35, \mathrm{P}=$ $0.05)$.

Finally, given previous reports that participation in exercise is associated with generation of natural ligands for the nuclear receptor protein peroxisome proliferator-activated receptor-gamma (PPAR $\gamma$ ) within the vasculature $[36,37]$, that pharmacological activators of the nuclear receptor PPAR $\gamma$ are associated with decreased MMP-9 secretion from human monocytes [38], and that PPAR $\gamma$ may bring about this effect by antagonistic transrepression at nuclear factor-kappa Beta response elements (NFkB-RE) and activator protein-1 sites [39, 40], we performed bioinformatics screens of the human MMP-9 promoter (accession code: NG_011468.1) for peroxisome proliferator response elements (PPRE), NFkB-RE and AP-1 consensus sequences. No PPREs were found within $\sim 5 \mathrm{Kbp}$ of the MMP-9 start site, but sequences were found resembling the reported consensus sequences for NFkB-RE [41] and AP-1 [42] at positions -4076bp, -637bp, -408bp, -349bp; and positions $-1680 \mathrm{bp},-556 \mathrm{bp}$, respectively.

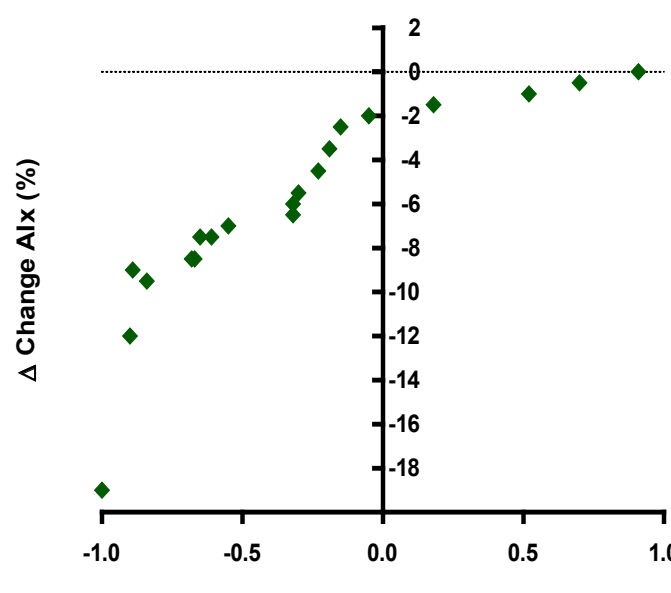

$\Delta$ Change MMP-9 Expression (cf. GAPDH)

Figure 5: The relationship between the changes in augmentation index and matrix metalloproteinase- 9 expression recorded between baseline and 8-weeks of a green exercise programme. A statistically significant association between measured changes in augmentation index (as determined using applanation tonometry) and in MMP-9 mRNA expression (as determined using RT-PCR) was identified $(r=0.87, P<0.001)$ using Pearson's correlation method. 
Citation: Thompson JES, Webb R, Hewlett P, Llewellyn D, McDonnell BJ (2013) Matrix Metalloproteinase-9 and Augmentation Index are Reduced with an 8-Week Green-Exercise Walking Programme. J Hypertens 2: 127. doi:10.4172/2167-1095.1000127

\section{Discussion}

This is the first study, to our knowledge, that has investigated the impact of a moderate-intensity, aerobic green-exercise programme on the mRNA expression of MMP-9 and markers of CV risk in a healthy cohort.

Our major novel finding illustrated a significant reduction in the expression of the MMP-9 gene following adherence to an exercise programme. The group that adhered to the exercise programme exhibited a significant reduction in the expression of MMP-9 over the 8-week period (compared to the non-adherent group) (Figure 3). Moreover, a statistically significant inverse correlation was observed between the change in MMP-9 mRNA expression and the change in the amount of exercise undertaken (as determined by IPAQ METminutes scores) (Figure 4); this illustrates that increasing levels of aerobic exercise in a healthy population is associated with a decrease in the expression of the MMP-9 gene. Further to this, a significant positive correlation was observed between this reduction in MMP-9 expression and a reduction in AIx. To our knowledge, this is the first time that the following two observations have been demonstrated: (i) an association between expression of MMP-9 and wave reflection; and (ii) that an aerobic, exercise-only intervention in a healthy, sedentary cohort has elicited a significant reduction in MMP-9 expression. Importantly, by identifying an association between MMP-9 expression and AIx, these data may provide a biomolecular mechanism to underpin our previous observational data, in which our group have illustrated that AIx was significantly lower in highly physically active young individuals compared to sedentary controls [3].

Interestingly, the lack of effect on anthropometric parameters such as waist circumference, BMI and body weight imply that the mechanism(s) triggered by the current intervention are distinct from weight loss per se (Table 3 ). Similarly, there was no change in aPWV observed in the present study. This was expected as McEniery et al. have already demonstrated that in adults less than $\sim 50$ years old, AIx is a more sensitive marker of arterial stiffening (and hence of CV risk), whereas aPWV is a better measure of stiffness in adults over $\sim 50$ years of age [43]. Further to this, an 8-week long programme of moderate-intensity exercise may not be a long enough period of time to elicit a change in the structure of the large arteries, with previous studies demonstrating that a moderate-intensity programme of at least four months or an 8 -week programme of high-intensity exercise is required in order to observe a reduction in aPWV [44,45]. Accordingly, previous data from our own group have demonstrated that in a young population, there is no difference in aPWV between young highly active individuals and young age-matched sedentary participants [3]. McDonnell et al. [3] also demonstrated that older participants ( $>50$ years old) who regularly participated in physical activity have lower aPWV (and therefore lower $\mathrm{CV}$ risk) compared to age-matched sedentary controls. Thus, understanding the underlying biomolecular mechanisms associated with these exercise-associated observations becomes very important.

Reductions in MMP-9 levels would result in a decrease in the breakdown of the elastin component of the ECM, and such reductions would therefore result in a blunting of age-related increases in arterial stiffness and remodelling of the vascular wall. In contrast, an inverse relationship between aPWV and serum levels of enzymatically active MMP-9 has previously been observed in a cross-sectional study [46]. However, it should be noted that in this study, physical activity data were not collected and hence differential levels of physical activity within the cohort may explain the observed inverse relationship. In the current study, enzymatically active MMP-9 was assessed in the plasma via ELISA, and exhibited a significant positive correlation with leukocytic MMP-9 mRNA levels. Therefore, we suggest based on the current data that the lower levels of stiffness discussed above occur as a result of continuous exercise-induced reductions in MMP-9, and therefore reductions in age-related increases in arterial stiffness. Clearly however, further experiments are required to confirm this suggestion.

A potential mechanism by which exercise impacts upon MMP9 expression is via the activation of $\operatorname{PPAR} \gamma$, which functions as a transcription factor regulating the expression of PPRE-bearing target genes in a ligand-dependent manner. Interestingly, the synthetic PPAR $\gamma$ ligand Rosiglitazone (RSG) has been shown to decrease both expression and activity of MMP-9 [39]. It has been reported that RSG-activated PPAR $\gamma$ may bring about this effect by antagonistic transrepression of a second transcription factor, namely NFkB, and our bioinformatics analyses support this view, in that several NFkB-REs, but not PPREs, were identified in the MMP-9 promoter [39].

The actions of Thiazolidinediones (TZDs) such as RSG in regulating PPAR $\gamma$ target gene expression have been shown to be responsible for beneficial effects in the context of CVD and Type-2 Diabetes [47], including decreasing arterial stiffness over a 12 -week period, in a diabetic population [48]. Further to this, Ryan et al. [49] and Hetzel et al. [50] have reported reductions in PWV and improvements in endothelial function in response to TZD treatment in non-diabetic cohorts. This suggests that the improvements observed in AIx in the current study (which suggest an improvement in endothelial function) may also occur as a result of exercise-induced activation of PPAR $\gamma$. Thus, while due to an association with increased risk of death from CV causes [51], the prescription of RSG has fallen dramatically in recent years [52], exercise may provide a safe alternative to RSG as a method of inducing beneficial PPAR $\gamma$-dependent effects $[36,37]$. Therefore, with regard to the current study's findings, we propose that an exerciseinduced activation of the nuclear receptor $\operatorname{PPAR} \gamma$ may cause the observed down-regulation in the expression of the elastin-degrading gene MMP-9 (and also possibly improve endothelial function), which in turn may ameliorate physical inactivity-related vascular remodelling.

The second novel finding of the current study is the demonstration that adherence specifically to an 8-week, moderate-intensity, aerobic green-exercise programme of a sedentary, healthy population is associated with significant reductions in several haemodynamic factors related to CV risk, namely MAP see (see Figure 1), AIx (as a measure of wave reflection; see Figure 2) and MMP-9 expression (see Figure 3). These reductions in MAP and AIx suggest an improvement in the functionality of the resistance arteries and therefore a reduction in $\mathrm{CV}$ risk. The results were novel (in the context specifically of green-exercise) but expected, as aerobic exercise programmes have previously been associated with improvements in CV risk and vascular haemodynamics, including MAP and AIx [53-55]. However, to the best of our knowledge, while Pretty et al. [21] have demonstrated the beneficial impact of acute bouts of aerobic exercise whilst viewing scenes of nature on MAP, the effect of an 8-week green-exercise programme on MAP and AIx had not before been investigated. Importantly, the beneficial impact of greenexercise walking programmes upon markers of CV risk is important from a public health/policy context, as green-exercise provides a route to exercise that overcomes most reported barriers to exercising, due to the fact that it is free, requires no special equipment or clothing, can be carried out at any age or fitness and is easily accessible $[21,23]$. 
Citation: Thompson JES, Webb R, Hewlett P, Llewellyn D, McDonnell BJ (2013) Matrix Metalloproteinase-9 and Augmentation Index are Reduced with an 8-Week Green-Exercise Walking Programme. J Hypertens 2: 127. doi:10.4172/2167-1095.1000127

Page 7 of 8

The current study has several limitations: for example, the current investigation needs to be replicated in a larger cohort (the correlation analyses in particular should be interpreted with caution due to the small sample size), and over a longer period of time in order to confirm the above findings. Furthermore, a longitudinal study would determine whether a long-term down-regulation of MMP-9 is associated with changes in aPWV. However, the authors would add that no confounding factors or outliers were identified in our datasets, and therefore we are reasonably confident in the validity of the conclusions arising from our analyses. Such future studies may provide additional evidence supporting the concept that exercise slows age-related arterial stiffness due to a reduction in MMP-9-catalysed elastin-degradation and therefore vascular remodelling. Moreover, further investigation of the role of PPAR $\gamma$ signalling in the effects investigated here should be carried out in order to confirm or disprove the suggestion that this signalling pathway has contributed to the effects seen in the current study. Nevertheless, despite these limitations, the current study was successful in meeting its aims and the current authors would contend that the study provides strong evidence that beneficial reductions in markers of CV risk can be triggered in sedentary participants who significantly increase their physical activity levels via means of a moderate-intensity, aerobic, unstructured green-exercise programme. As stated earlier, most barriers reported as reasons not to exercise apply to a much lesser extent with regard to green-exercise, making it a potential route by which participation in exercise may be increased, and therefore possibly a useful tool by which physical activity levels can be increased and CV risk decreased within the general population [23].

\section{Clinical relevance}

The current study demonstrates that a potential mechanism for the lowering of large artery stiffness and CV risk is via green-exercise programmes. Its findings suggest that one mechanism underlying this reduction in risk may occur via an exercise-associated reduction in the expression of the elastin-degrading enzyme, MMP-9. The observed association between decreases in MMP-9 and wave reflection (as measured via AIx) suggest that MMP-9 may have a role to play in the progression of the early return of the reflected wave, and therefore in increases in central systolic pressure. Thus, if exercise decreases the expression of the enzymes that are involved in age-related increases in vascular remodelling and large artery stiffness, then progression of increased systolic hypertension may be significantly reduced as a consequence.

\section{Conclusions}

Thus, in conclusion, the findings presented in the current study may provide a biomolecular mechanism (namely, exercise-associated MMP-9 downregulation) that contributes towards aerobic exercise's ability to delay age-related increases in wave reflection, large artery stiffness and therefore in CV risk. Moreover, we suggest that low SESassociated physical inactivity and CV risk may be combated using free and easily accessible green-exercise programmes.

\section{Acknowledgement}

We would like to acknowledge the laboratory staff at the Diabetes Research Network Wales laboratories (Swansea University) for their contribution in the running of the biochemical analyses.

\section{Source of Funding}

Ms Thompson is the beneficiary of a Ph.D. scholarship funded by the European Social Fund's Knowledge Enterprise Skills Scholarship, with the collaboration and support of Groundwork Wales Ltd and Cardiff Metropolitan University $(\sim £ 102,000)$. Some of the data in this paper were presented at the 23rd Annual Meeting of the
European Society of Hypertension (June, 2013)

\section{References}

1. Kotsis V, Stabouli S, Karafillis I, Nilsson $P$ (2011) Early vascular aging and the role of central blood pressure. J Hypertens 29: 1847-1853.

2. Vaitkevicius PV, Fleg JL, Engel JH, O'Connor FC, Wright JG, et al. (1993) Effects of age and aerobic capacity on arterial stiffness in healthy adults. Circulation 88: 1456-1462.

3. McDonnell BJ, Maki-Petaja KM, Munnery M, Yasmin, Wilkinson IB, et al (2013) Habitual exercise and blood pressure: age dependency and underlying mechanisms. Am J Hypertens 26: 334-341.

4. Sugawara J, Otsuki T, Tanabe T, Hayashi K, Maeda S, et al. (2006) Physical activity duration, intensity, and arterial stiffening in postmenopausal women. Am J Hypertens 19: 1032-1036.

5. Safar ME, Levy BI, Struijker-Boudier H (2003) Current perspectives on arteria stiffness and pulse pressure in hypertension and cardiovascular diseases. Circulation 107: 2864-2869.

6. Sutton-Tyrrell K, Najjar SS, Boudreau RM, Venkitachalam L, Kupelian V, et al. (2005) Elevated aortic pulse wave velocity, a marker of arterial stiffness predicts cardiovascular events in well-functioning older adults. Circulation 111 3384-3390.

7. Lakatta EG (2003) Arterial and cardiac aging: major shareholders in cardiovascular disease enterprises: Part III: cellular and molecular clues to heart and arterial aging. Circulation 107: 490-497.

8. Safar ME (1999) Hypothesis on isolated systolic hypertension in the elderly. J Hum Hypertens 13: 813-815.

9. Galis ZS, Khatri JJ (2002) Matrix metalloproteinases in vascular remodeling and atherogenesis: the good, the bad, and the ugly. Circ Res 90: 251-262.

10. Handa S, Sadi AM, Cybulsky MI, Stewart DJ, Husain M (2008) Region-specific patterns of vascular remodelling occur early in atherosclerosis and without loss of smooth muscle cell markers. Atherosclerosis 196: 617-623.

11. Cho A, Reidy MA (2002) Matrix metalloproteinase-9 is necessary for the regulation of smooth muscle cell replication and migration after arterial injury. Circ Res 91: 845-851.

12. Greenwald SE (2007) Ageing of the conduit arteries. J Pathol 211: 157-172.

13. Liao D, Arnett DK, Tyroler HA, Riley WA, Chambless LE, et al. (1999) Arteria stiffness and the development of hypertension. The ARIC study. Hypertension 34: 201-206

14. Nagase H, Woessner JF Jr (1999) Matrix metalloproteinases. J Biol Chem 274 21491-21494

15. Brew K, Dinakarpandian D, Nagase H (2000) Tissue inhibitors of metalloproteinases: evolution, structure and function. Biochim Biophys Acta1477: 267-283.

16. Yasmin, McEniery CM, Wallace S, Dakham Z, Pulsalkar P, et al. (2005) Matrix metalloproteinase-9 (MMP-9), MMP-2, and serum elastase activity are associated with systolic hypertension and arterial stiffness. Arterioscler Thromb Vasc Biol 25: 372.

17. Laurent S, Cockcroft J, Van Bortel L, Boutouyrie P, Giannattasio C, et al. (2006) Expert consensus document on arterial stiffness: methodological issues and clinical applications. Eur Heart J 27: 2588-2605.

18. Tanaka H, DeSouza CA, Seals DR (1998) Absence of age-related increase in central arterial stiffness in physically active women. Arterioscler Thromb Vasc Biol 18: 127-132.

19. Tanaka H, Dinenno FA, Monahan KD, Clevenger CM, DeSouza CA et al. (2000) Aging, habitual exercise, and dynamic arterial compliance. Circulation 102: 1270-1275.

20. Farrell L, Hollingsworth B, Propper C, Shields MA (2013) The Socioeconomic Gradient in Physical Inactivity in England, in The Centre For Market And Public Organisation.

21. Pretty J, Peacock J, Sellens M, Griffin M (2005) The mental and physical health outcomes of green exercise. Int J Environ Health Res 15: 319-337.

22. Kaplan S (1995) The restorative benefits of nature: Toward an integrative framework. J Environ Psychol 15: 169-182.

23. Salmon J, Owen N, Crawford D, Bauman A, Sallis JF (2003) Physical activity 
Citation: Thompson JES, Webb R, Hewlett P, Llewellyn D, McDonnell BJ (2013) Matrix Metalloproteinase-9 and Augmentation Index are Reduced with an 8-Week Green-Exercise Walking Programme. J Hypertens 2: 127. doi:10.4172/2167-1095.1000127

and sedentary behavior: a population-based study of barriers, enjoyment, and preference. Health Psychol 22: 178-188.

24. Cook MD, Heffernan KS, Ranadive S, Woods JA, Fernhall B (2013) Effect of resistance training on biomarkers of vascular function and oxidative stress in young African-American and Caucasian men. J Hum Hypertens 27: 388-392.

25. Roberts CK, Won D, Pruthi S, Kurtovic S, Sindhu RK, et al. (2006) Effect of a short-term diet and exercise intervention on oxidative stress, inflammation, MMP-9, and monocyte chemotactic activity in men with metabolic syndrome factors. J Appl Physiol (1985) 100: 1657-1665.

26. Roberts CK, Chen AK, Barnard RJ (2007) Effect of a short-term diet and exercise intervention in youth on atherosclerotic risk factors. Atherosclerosis 191: 98-106

27. Kadoglou NP, Vrabas IS, Sailer N, Kapelouzou A, Fotiadis G, et al. (2010) Exercise ameliorates serum MMP-9 and TIMP-2 levels in patients with type 2 diabetes. Diabetes Metab 36: 144-151.

28. Motl RW, Konopack JF, McAuley E, Elavsky S, Jerome GJ, et al. (2005) Depressive symptoms among older adults: long-term reduction after a physical activity intervention. J Behav Med 28: 385-394.

29. Huggins R, Thompson P, UK Competitiveness Index, Centre for International Competitiveness, Cardiff School of Management, University of Wales Institute, Cardiff.

30. Haskell WL, Lee IM, Pate RR, Powell KE, Blair SN, et al. (2007) Physica activity and public health: updated recommendation for adults from the American College of Sports Medicine and the American Heart Association. Med Sci Sports Exerc 39: 1423-1434

31. O'Brien E, Asmar R, Beilin L, Imai Y, Mallion JM, et al. (2003) European Society of Hypertension recommendations for conventional, ambulatory and home blood pressure measurement. J Hypertens 21: 821-848.

32. Benetos A, Safar M, Rudnichi A, Smulyan H, Richard JL, et al. (1997) Pulse pressure: a predictor of long-term cardiovascular mortality in a French male population. Hypertension 30: 1410-1415.

33. Friedewald WT, Levy RI, Fredrickson DS (1972) Estimation of the concentration of low-density lipoprotein cholesterol in plasma, without use of the preparative ultracentrifuge. Clin Chem 18: 499-502.

34. R\&D Systems, Quantikine ELISA HUMAN MMP-9, in Tools for Cell Biology Research, Abingdon, UK.

35. Kadoglou NP, Daskalopoulou SS, Perrea D, Liapis CD (2005) Matrix metalloproteinases and diabetic vascular complications. Angiology 56: 173189.

36. Butcher LR, Thomas A, Backx K, Roberts A, Webb R, et al. (2008) Low-intensity exercise exerts beneficial effects on plasma lipids via PPARgamma. Med Sci Sports Exerc 40: 1263-1270.

37. Thomas AW, Davies NA, Moir H, Watkeys L, Ruffino JS, et al. (2012) Exerciseassociated generation of PPAR $\hat{l}^{3}$ ligands activates PPAR $\hat{l}^{3}$ signaling events and upregulates genes related to lipid metabolism. J Appl Physiol (1985) 112: 806-815.

38. Shu H, Wong B, Zhou G, Li Y, Berger J, et al. (2000) Activation of PPARa Reduces Secretion of Matrix Metalloproteinase 9 but Not Interleukin 8 from Human Monocytic THP-1 Cells. Biochem Biophys Res Commun 267: 345-349.

39. Hetzel M, Walcher D, Grüb M, Bach H, Hombach V, et al. (2003) Inhibition of MMP-9 expression by PPARgamma activators in human bronchial epithelial cells. Thorax 58: 778-783.

40. Schroen DJ, Brinckerhoff CE (1996) Nuclear hormone receptors inhibit matrix metalloproteinase (MMP) gene expression through diverse mechanisms. Gene Expr 6: 197-207.

41. Novak U, Cocks BG, Hamilton JA (1991) A labile repressor acts through the NFkB-like binding sites of the human urokinase gene. Nucleic Acids Res 19 3389-3393.
42. Lee W, Mitchell P, Tjian R (1987) Purified transcription factor AP-1 interacts with TPA-inducible enhancer elements. Cell 49: 741-752.

43. McEniery CM, Yasmin, Hall IR, Qasem A, Wilkinson IB, et al. (2005) Normal vascular aging: differential effects on wave reflection and aortic pulse wave velocity: the Anglo-Cardiff Collaborative Trial (ACCT). J Am Coll Cardiol 46: 1753-1760.

44. Hayashi K, Sugawara J, Komine H, Maeda S, Yokoi T (2005) Effects of aerobic exercise training on the stiffness of central and peripheral arteries in middleaged sedentary men. Jpn J Physiol 55: 235-239.

45. Kakiyama T, Sugawara J, Murakami H, Maeda S, Kuno S, et al. (2005) Effects of short-term endurance training on aortic distensibility in young males. Med Sc Sports Exerc 37: 267-271.

46. Vlachopoulos C, Aznaouridis K, Dima I, loakeimidis N, Vasiliadou C, et al. (2007) Negative association between serum levels of matrix metalloproteinases- 2 and -9 and aortic stiffness in healthy adults. Int J Cardiol 122: 232-238.

47. DREAM (Diabetes REduction Assessment with ramipril and rosiglitazone Medication) Trial Investigators, Gerstein HC, Yusuf S, Bosch J, Pogue J, et al. (2006) Effect of rosiglitazone on the frequency of diabetes in patients with impaired glucose tolerance or impaired fasting glucose: a randomised controlled trial. Lancet 368: 1096-1105.

48. Yu J, Jin N, Wang G, Zhang F, Mao J, et al. (2007) Peroxisome proliferatoractivated receptor gamma agonist improves arterial stiffness in patients with type 2 diabetes mellitus and coronary artery disease. Metabolism 56: 13961401.

49. Ryan KE, McCance DR, Powell L, McMahon R, Trimble ER (2007) Fenofibrate and pioglitazone improve endothelial function and reduce arterial stiffness in obese glucose tolerant men. Atherosclerosis 194: e123-130.

50. Hetzel J, Balletshofer B, Rittig K, Walcher D, Kratzer W, et al. (2005) Rapid effects of rosiglitazone treatment on endothelial function and inflammatory biomarkers. Arterioscler Thromb Vasc Biol 25: 1804-1809.

51. Nissen SE, Wolski K (2007) Effect of rosiglitazone on the risk of myocardia infarction and death from cardiovascular causes. N Engl J Med 356: 2457 2471.

52. Leal I, Romio SA, Schuemie M, Oteri A Sturkenboom M, et al. (2013) Prescribing pattern of glucose lowering drugs in the United Kingdom in the last decade: a focus on the effects of safety warnings about rosiglitazone. $\mathrm{Br} \mathrm{J}$ Clin Pharmacol 75: 861-868.

53. Goldberg MJ, Boutcher SH, Boutcher YN (2012) The effect of 4 weeks of aerobic exercise on vascular and baroreflex function of young men with a family history of hypertension. J Hum Hypertens 26: 644-649.

54. Edwards DG, Schofield RS, Magyari PM, Nichols WW, Braith RW (2004) Effect of exercise training on central aortic pressure wave reflection in coronary artery disease. Am J Hypertens 17: 540-543.

55. Figueroa A, Park SY, Seo DY, Sanchez-Gonzalez MA, Baek YH (2011) Combined resistance and endurance exercise training improves arterial stiffness, blood pressure, and muscle strength in postmenopausal women. Menopause 18: 980-984. 